Revue musicale OICRM

\title{
Mélodie et orientalisme
}

\section{De l'évocation du merveilleux aux séductions de l'avant-garde}

\section{Sylvain Caron}

Volume 3, numéro 1, 2016

Musique et exotisme en France au tournant du XXe siècle. Altérités recomposées

URI : https://id.erudit.org/iderudit/1060123ar

DOI : https://doi.org/10.7202/1060123ar

Aller au sommaire du numéro

\section{Éditeur(s)}

OICRM

ISSN

2368-7061 (numérique)

Découvrir la revue

Citer cet article

Caron, S. (2016). Mélodie et orientalisme : de l'évocation du merveilleux aux séductions de l'avant-garde. Revue musicale OICRM, 3(1), 93-114.

https://doi.org/10.7202/1060123ar

\section{Résumé de l'article}

Au tournant du XXe siècle, les mélodies françaises représentent l'Orient comme un ailleurs merveilleux. Elles déclenchent un voyage imaginaire par un jeu de figures musicales évoquant souvent un univers riche en stimulations sensorielles (Les roses d'Ispahan de Leconte de Lisle/Fauré) ou en symbolisme érotisme (Trois chansons de Bilitis de Louÿs/Debussy). Or, dans la seconde décennie du siècle, l'Orient perd son aura évocatrice ; il devient plutôt l'élément déclencheur d'explorations avant-gardistes. Nourris par les découvertes musicologiques et par le développement des moyens de transport, les compositeurs deviennent plus sensibles à l'acte même de composer dans les cultures extra-européennes et au rapport au son qu'il implique. En choisissant le timbre musical comme moteur de la forme, ils créent de nouveaux paysages musicaux. C'est ainsi que les Quatre poèmes hindous de Delage et les Chansons madécasses de Ravel s'approprient l'Orient comme la source pure d'une humanité retrouvée, gage d'une authenticité artistique véritable. 


\title{
Mélodie et orientalisme. De l'évocation du merveilleux aux séductions de l'avant-garde
}

\author{
Sylvain Caron
}

\section{Résumé}

$\mathrm{Au}$ tournant $\mathrm{du} \mathrm{xx}^{\mathrm{e}}$ siècle, les mélodies françaises représentent l'Orient comme un ailleurs merveilleux. Elles déclenchent un voyage imaginaire par un jeu de figures musicales évoquant souvent un univers riche en stimulations sensorielles (Les roses d'Ispahan de Leconte de Lisle/Fauré) ou en symbolisme érotisme (Trois chansons de Bilitis de Louÿs/Debussy). Or, dans la seconde décennie du siècle, l'Orient perd son aura évocatrice ; il devient plutôt l'élément déclencheur d'explorations avantgardistes. Nourris par les découvertes musicologiques et par le développement des moyens de transport, les compositeurs deviennent plus sensibles à l'acte même de composer dans les cultures extra-européennes et au rapport au son qu'il implique. En choisissant le timbre musical comme moteur de la forme, ils créent de nouveaux paysages musicaux. C'est ainsi que les Quatre poèmes hindous de Delage et les Chansons madécasses de Ravel s'approprient l'Orient comme la source pure d'une humanité retrouvée, gage d'une authenticité artistique véritable.

Mots clés : avant-garde ; mélodie française ; musique française ; orientalisme ; peinture et musique.

\begin{abstract}
At the turn of the 20th century, French mélodies portray the Middle East as a faraway and marvellous wonderland. Through a brilliant setting of musical figures, they generate an imaginary journey evocating a luxuriant world of either sensory stimulations (Les Roses d'Ispahan by Leconte de Lisle/Fauré) or erotic symbolism (Trois Chansons de Bilitis by Louÿs/Debussy). During the second decade of the century, though, its suggestive strength went weak; it became rather a trigger for vanguard experimentations. Nourished by musicological discoveries and the development of public passenger transport systems, musicians are becoming increasingly sensitive towards the act of composing in extra-European cultures and its relation with sound. By choosing musical timbre as cornerstone for the composition form, they create new musical landscapes. Thus, Delage's Quatre Poèmes Hindous and Ravel's Chansons Madécasses take the Middle East as the utter root of a rediscovered humanity, pledge of a true artistic authenticity.
\end{abstract}

Keywords: avant-garde; French mélodie; French music; orientalism; painting and music. 
Les principales idées de cet article ont d'abord été présentées lors d'une conférence au Musée des beaux-arts de Montréal, dans le cadre de l'exposition "Merveilles et mirages de l'orientalisme ". Les deux commissaires de l'exposition, Nathalie Bondil et Axel Hémery, mentionnent dans la préface du catalogue que l'Orient de Benjamin-Constant a été déclenché par un voyage à Grenade et à Tanger, qui a agi sur l'imaginaire du peintre : " ethnographie tirée vers le pittoresque, fascination sulfureuse de la femme orientale fantasmée, peinture d'histoire ponctuée de violences et d'arbitraire " (Bondil 2014, p. 21). Un peu plus loin, dans le chapitre consacré à "La palette du peintre ", Hémery ajoute que le tableau intitulé Entrée du sultan Mehmet II à Constantinople le 29 mai 1453 (1876 ; figure 1) « n'est pas la première œuvre orientaliste de Benjamin-Constant, mais [que] c'est celle qui bénéficia du plus grand retentissement et qui lancera le débat sur la nature de l'art de coloriste du peintre » (dans ibid., p. 30). Voulant se démarquer des représentations traditionnelles, le peintre prend appui sur l'orientalisme pour affirmer l'originalité de sa démarche coloriste. Rétrospectivement, lorsqu'on regarde l'importance que prend la couleur chez les peintres $d u$ tournant $d u x^{e}$ siècle, cette démarche prend valeur de "symptôme culturel », au sens où l'emploi Ernst Gombrich dans son Histoire de l'art (2001).

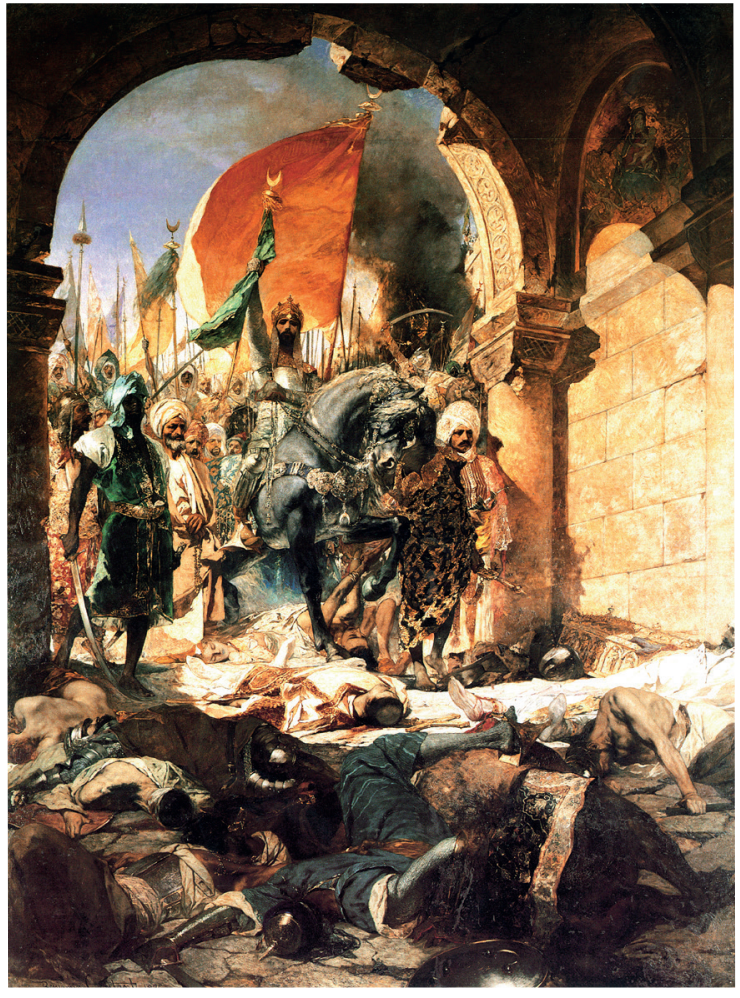

Figure 1: Benjamin-Constant, Entrée du sultan Mehmet II à Constantinople le 29 mai 1453, 1876. Huile sur toile, $697 \times 536 \mathrm{~cm}$. Toulouse, Musée des Augustins. Source: Art Renewal Center Museum, image 10603, sous licence Domaine public via Wikimedia Commons.

En musique, peut-on retrouver chez des compositeurs français de la même période une démarche analogue ? Oui, et en particulier dans la mélodie, un genre destiné à un public érudit et qui n'est pas soumis à l'impératif d'une réussite auprès du grand public comme peut l'être l'opéra. Ce type de questionnement interdisciplinaire soulève toutefois une question méthodologique : comment articuler de manière féconde, musicologiquement, un discours comparatiste entre les techniques de création picturales et 
musicales? Dans La musique, les images et les mots (2010), Jean-Jacques Nattiez montre comment la comparaison des stratégies de création permet d'enrichir et de documenter la réflexion en esthétique comparée, tout en préservant par ailleurs le discours de la " tentation analogique ${ }^{1}$ ". La perspective dans laquelle s'inscrivent les études interdisciplinaires entre peinture et musique se concentre souvent sur les œuvres ${ }^{2}$. Dans cet article, nous chercherons plutôt à nous concentrer sur la transférabilité des méthodes et des stratégies de création : en quoi une théorie émanant du domaine de la peinture peut-elle nous permettre d'approcher différemment la musique?

Les historiens de l'art - notamment Nicole Dubreuil, avec qui nous avons discuté en préparant cet article - citent souvent une phrase de Maurice Denis, afin de décrire l'évolution de la peinture au tournant $\mathrm{du} \mathrm{xx}^{\mathrm{e}}$ siècle : "Se rappeler qu'un tableau - avant d'être un cheval de bataille, une femme nue, ou une quelconque anecdote - est essentiellement une surface plane recouverte de couleurs en un certain ordre assemblées » (Denis 1914, p. 1). Même s'il est très souvent cité, cet aphorisme n'en demeure pas moins une phrase-clé dans les théories picturales du tournant du $\mathrm{XX}^{\mathrm{e}}$ siècle. D'une part, Denis y affirme la matérialité de l'œuvre, avec ses formes, ses couleurs, sa surface, alors que d'autre part, il insiste sur la nécessité d'organiser la surface picturale selon un principe d'ordre. Il adopte une logique positiviste voulant que le signe porte en lui-même sa propre expression, indépendamment de son renvoi à une réalité extérieure (Bouillon 2006, p. 19). Certains tableaux des années 1890, comme Taches de soleil sur la terrasse (figure 2), sont plus fortement tournés vers une expression des formes et des couleurs prises pour elles-mêmes. Dans son essai sur les origines positivistes du mouvement nabi, Jean-Paul Bouillon explique que Denis s'est inspiré du chapitre IV, quatrième sous-chapitre, de la Philosophie de l'art d'Hippolyte Taine (Taine [1882]1985), pour formuler sa définition :

Par elles-mêmes et en dehors de leur emploi imitatif, les couleurs, comme les lignes, ont un sens. Une gamme de couleurs qui ne figurent aucun objet réel, comme une arabesque de lignes qui n'imitent aucun objet naturel, peut être riche ou maigre, élégante ou lourde. Notre impression varie avec leur assemblage ; leur assemblage a donc une expression. Un tableau est une surface colorée, dans laquelle les divers tons et les divers degrés de lumière sont répartis avec un certain choix ; voilà son être intime, que ces tons et ces degrés de lumière fassent des figures, des draperies, des architectures, c'est là pour eux une propriété ultérieure, qui n'empêche pas leur propriété primitive d'avoir toute son importance et tous ses droits (ibid., p. 452-453).

$1 \quad$ Voir aussi Nattiez 2008.

2 Voir, par exemple, Nattiez 2010, Junod 2006 et Barbe 2011. 


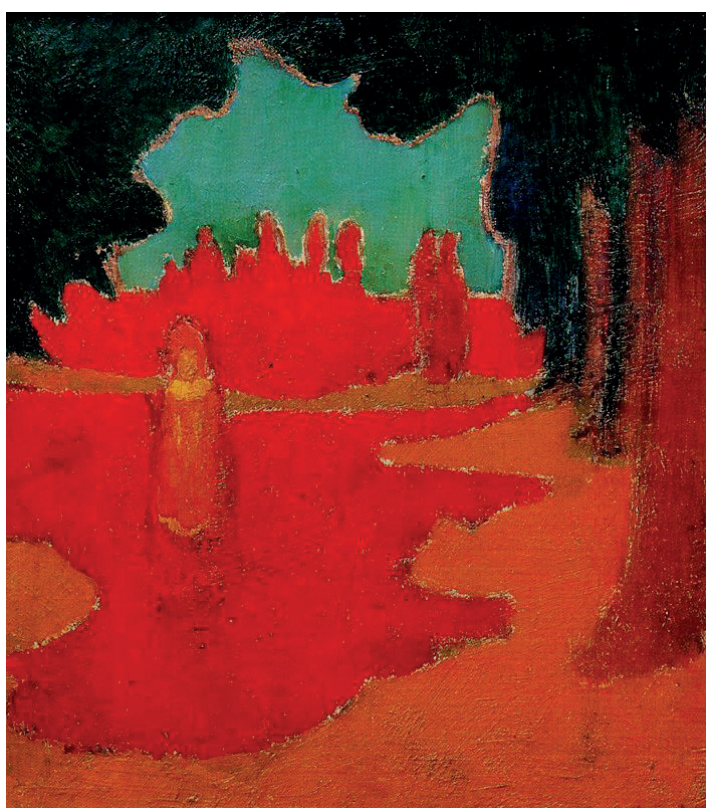

Figure 2 : Maurice Denis, Taches de soleil sur la terrasse, 1890. Huile sur carton, $24 \times 20,5 \mathrm{~cm}$. Paris, Musée d'Orsay. Source : sous licence Domaine public via WikiArt.

On pourrait objecter avec raison que, pour parler d'orientalisme, il faut bien évoquer un ailleurs et non se limiter aux lois qui régissent la matérialité de l'œuvre. De fait, Denis n'a pas renoncé à mettre en peinture des récits, comme dans les panneaux de La légende de Saint-Hubert (figure 3). Ce maintien d'une portée narrative dans ses peintures nous permet de nuancer la citation de Denis : celui-ci établit une sorte de dialogue entre ce qui est évoqué par le récit et la matérialité du tableau. Sa démarche artistique vise à toucher l'esprit par la forme et les couleurs, par les spécificités du médium, tant que par le contenu ou le lien narratif.

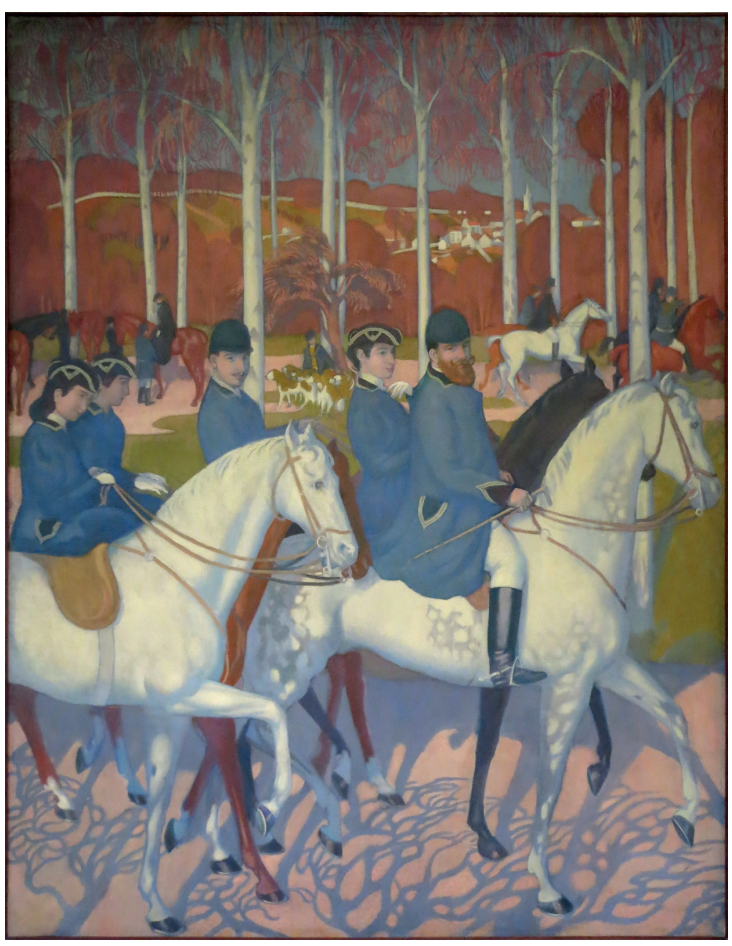

Figure 3 : Maurice Denis, La légende de SaintHubert. Le départ (extrait d'un ensemble de sept panneaux), 1897. Huile sur toile, $225 \times 175 \mathrm{~cm}$. Saint-Germain-en-Laye, musée départemental Maurice Denis. Source : sous licence Domaine public via Wikimedia Commons. 
Nous devons enfin préciser ce que nous entendons par l'appellation « orientalisme». $\mathrm{Au}$ sens strict, Locke la définit comme un "dialecte » de l'exotisme musical au sein de la tradition occidentale, qui évoque l'Est ou l'Orient. Au sens large, elle évoque un ailleurs englobant le Moyen-Orient, l'Afrique du Nord et l'Asie (Locke 2009). Dans l'univers de Benjamin-Constant, l'Espagne mauresque et même la Constantinople antique sont incluses dans la notion d'orientalisme. L'idée de l'Orient peut aussi englober la Grèce antique, dans ce qu'elle comporte de mythologique (comme chez Debussy). Dans cet article, nous abordons la question de l'orientalisme musical dans la perspective positiviste de Taine ${ }^{3}$ et de Denis. La mélodie française évolue dans une direction analogue à celle prophétisée par le jeune $\mathrm{Nabi}^{4}$, en ce sens que le thème de l'orientalisme évolue à partir d'une représentation dix-neuviémiste, basée sur le merveilleux ou sur la sensualité érotisante de la femme, en allant vers une expression où un élément orientalisant est délibérément abstrait de son contexte d'origine ${ }^{5}$, et qu'ainsi libéré d'une fonction d'évocation conventionnelle il puisse nourrir des formes d'expressions tournées vers 1'avant-garde. En paraphrasant Denis, nous pourrions dire qu'avant d'être une scène orientale, une évocation de la sensualité féminine ou une danse exotique, une musique est essentiellement un intervalle de temps où l'on entend des timbres, des mélodies et des rythmes en un certain ordre assemblés. La réflexion des compositeurs avant-gardistes, bien que n'excluant pas les dimensions narratives ou évocatrices, se tourne en premier lieu vers les propriétés de la musique elle-même et l'orientalisme sert à enrichir la démarche créative du compositeur en renouvelant son langage, en tirant de l'altérité un pouvoir de mise à distance de techniques de composition devenues traditionnelles.

$\mathrm{Au}$ cours des dernières années, la théorisation de la notion d'exotisme musical s'est enrichie d'un article de Jean-Pierre Bartoli intitulé : "Propositions pour une définition de l'exotisme musical et pour une application en musique de la notion d'isotopie sémantique », où l'auteur précise :

on désigne par exotisme un ensemble de procédures qui consiste à évoquer l'altérité culturelle et géographique. [L'exotisme] consiste le plus souvent à faire l'emploi d'unités significatives qui semblent ne pas appartenir au langage artistique idiomatique, mais paraissent empruntées à un langage artistique étranger (Bartoli 2000, p. 65).

3 Le mot positivisme doit être compris dans le sens de « doctrine qui se réclame de la seule connaissance des faits, de l'expérience scientifique » (Le Grand Robert, 2009). Dans sa Philosophie de l'art, Taine précise à plusieurs moments sa pensée positiviste. Ainsi, il écrit : "Qu'est-ce que l'Art, et en quoi consiste sa nature ? - Au lieu de vous imposer une formule, je vais vous faire toucher des faits. Car il y a des faits ici comme ailleurs, des faits positifs et qui peuvent être observés, j'entends des ceuvres d'art rangées par famille dans les musées et les bibliothèques, comme les plantes dans un herbier et les animaux dans un muséum » (Taine [1882]1985, p. 20). Plus loin, dans la cinquième partie, Taine explique sa conception de l'idéal dans l'art, appliquant les catégories des sciences naturelles à l'étude des œuvres d'art (ibid., p. 373-460).

$4 \quad$ Pour le groupe de peintres réuni autour de Denis, le mot nabi signifie prophète.

5 Sur cet emploi du verbe abstraire au sens de décontextualiser, voir Roque 2003, p. 92-118. 
Bartoli ajoute « qu'un paramètre isolé ne peut suffire à produire le sentiment de l'exotisme, la détermination du répertoire évoqué repose sur un assemblage de figures exotisantes "(Bartoli 2000, p. 64). Effectivement, le processus d'évocation musicale repose sur des informations que l'auditeur peut mettre en série, sur des figures musicales reconnues comme exotiques, ou d'un autre temps. Nous pouvons même ajouter, à la suite de Ralph Locke, que lorsque la musique fait référence à un texte littéraire, comme dans un opéra, le contexte des significations est encore plus global (Locke 2007, p. 477-521).

Pour l'exotisme du XIX ${ }^{\mathrm{e}}$ siècle, les approches de Bartoli et de Locke s'appliquent avec facilité. Orientées vers le domaine des significations exotisantes, ces définitions présupposent que l'œuvre guide l'auditeur vers un ailleurs réel ou imaginé. Ce qui est évoqué, ce sont des paysages, des tapisseries ou une représentation de la sensualité féminine, par exemple. Mais au $\mathrm{xx}^{\mathrm{e}}$ siècle, les compositeurs se tournent vers une démarche où la musique est surtout autoréférentielle. Au lieu de référer à une situation extérieure, la musique se réfère à elle-même, comme phénomène sonore. C'est dans la recherche de cette modernité que la citation de Denis peut nous guider. Comment concilier la quête de la sonorité en elle-même avec l'évocation d'un ailleurs oriental ? Est-ce simplement une affaire de titre ou de sujet poétique ? Et dans une avant-garde qui, par définition, fuit les conventions, le compositeur peut-il encore se permettre de puiser dans un bassin convenu de figures exotisantes, sans pour autant renier sa modernité?

Pour répondre à ces questions, un parcours à travers quelques mélodies peut nous éclairer. Fondamentalement, la mélodie est la mise en musique d'un poème. Les mots employés et les univers évoqués doivent assurément puiser dans les codes préexistants de l'exotisme, mais un poème n'est pas un récit ni un livret d'opéra ; c'est plutôt un court instant où se concentre une idée, envisagée sous des angles complémentaires. La mélodie française est un genre où, tacitement, l'auditeur consent à entrer dans le registre de la métaphore, voire du symbole, et aussi dans le registre de la sonorité des mots, ne serait-ce qu'à cause des rimes et des assonances. La forme poétique, avec ses vers, ses strophes et sa métrique, entre en dialogue avec la forme musicale. Dès lors, le registre expressif $d u$ poème est beaucoup plus tourné vers des réalités abstraites, vers l'art pris pour lui-même, que ne l'est l'opéra. C'est pourquoi la mélodie est un laboratoire pour plusieurs des compositeurs d'avant-garde ; et elle représente un angle intéressant pour aborder la question de l'orientalisme d'avant-garde au tournant $\mathrm{du} \mathrm{xx}^{\mathrm{e}}$ siècle, car elle met en action une série de paramètres musicaux et littéraires exotiques. À travers un parcours évolutif de mélodies composées par Fauré, Delage, Ravel et Debussy (figure 4), nous chercherons à identifier l'évolution des différents modes d'expression de l'Orient, afin de mieux saisir les enjeux qu'ils représentent pour l'avant-garde au début du $\mathrm{Xx}^{\mathrm{e}}$ siècle. 
- Gabriel Fauré, Les roses d'Ispahan, op. 39, n 4 (publiée en 1885), sur un poème de Leconte de Lisle ;

- Maurice Delage, "Lahore ", tirée des Quatre poèmes hindous (publiés en 1914), sur des poèmes de Bhartrihari, Heinrich Heine et d'un anonyme ;

- Maurice Ravel, « Nahandove », tirée des Chansons madécasses (publiées en 1926), sur des poèmes d'Évariste de Parny ;

- Claude Debussy, «La flûte de Pan ", tirée des Trois chansons de Bilitis (publiées en 1899), sur des poèmes de Pierre Louÿs ;

- Claude Debussy, «Le Faune », tirée des Fêtes galantes, vol. 2 (publiées en 1904) sur un poème de Paul Verlaine.

Figure 4: Tableau des mélodies étudiées.

LES Roses D'ISPAHAN, OP. 39, № 4 (1885), DE GabRIEl FaURÉ

En 1884, le compositeur Gabriel Fauré met en musique « Les roses d'Ispahan » du poète Leconte de Lisle. Nommer Ispahan dans le titre, c'était déjà faire référence à un réseau d'images, à l'une des cités les plus luxuriantes de la Perse ancienne, chargée de sensualité et de palais somptueux. Dans ce lieu, la femme occupe une double fonction, celle de corps sensuel et celle de muse inspiratrice. Comment la musique peut-elle, parallèlement aux mots du poème, parvenir à évoquer ces images ? Dans le contexte où vivait Fauré, il était d'usage que les poèmes récents soient d'abord proférés avant d'être lus. Selon cette pratique, le poème est d'abord une sonorité qui prend naissance sur les lèvres qui le prononcent. Entre ces lèvres passe le souffle qui est parole, ou encore, dans une mélodie, la voix chantée. Ce souffle est ressenti dans la proximité d'un petit espace comme le salon, et non dans une grande salle. Chez la cantatrice, la musique conditionne les inflexions du souffle. Selon les mots que l'on retrouve dans le poème "Le roses d'Ispahan ", le souffle est d'abord léger et doux, puis il devient intense lorsque le baiser est nommé. L'air se charge de la sensualité que dégage le parfum de la rose et de l'oranger. Dans la musique qui porte ces mots, c'est bien la nature vibratoire de la musique qui se manifeste, évoquant le battement d'aile d'un oiseau, animé par le mouvement quasi imperceptible de l'air déplacé par un papillon. Le poème, la musique et les interprètes sont bien présents dans l'œuvre telle qu'elle est entendue, ils entretiennent un jeu de circulation entre la matérialité et le travail de l'esprit, ce qui est une stratégie de création analogue à la perspective positiviste de Denis.

À la fois compositeur et interprète au piano de ses propres mélodies, Fauré est sensible à ces divers niveaux de réalité. Le choix qu'opère Fauré dans les strophes du poème (voir en annexe), n'en retenant que quatre sur six, va dans le sens d'une idéalisation de la nature féminine. La légèreté suspecte des amours de Leillah et le regret que réveille le souvenir de ses charmes sont supprimés ; la mélodie se concentre sur l'idéal d'un présent qui tient tous les sens en éveil.

La sensualité qui se dégage des Roses d'Ispahan a frappé les contemporains de Fauré, comme nous pouvons le lire dans La Revue musicale du 15 décembre 1906 : «Fauré a 
surtout vu là une occasion de séduire par des moyens un peu sensuels : ce n'est pas la pensée seule qui conquiert l'auditeur; on est pris comme dans un réseau de volupté où l'intelligence s'endort et où l'instinct est très doucement flatté... " (… B. 1906, p. 575) ${ }^{6}$. Ispahan agit davantage comme une toile de fond que comme le lieu de l'action. Ispahan déclenche une image de sensualité qui se détache de sa localisation géographique. La sensualité vient du caractère intime de la voix et du piano, de leur nuance douce, du rythme de danse langoureux. Si l'Orient séduit, ce n'est pas tant pour y voyager que pour en rêver. En cette fin de siècle, il n'est plus porté par les récits de voyage de Lamartine ${ }^{7}$, mais plutôt habité par l'imaginaire parnassien de Leconte de Lisle, que la musique de Fauré contribue à exalter. Au final, la nostalgie qui se dégage des Roses d'Ispahan n'est pas celle d'un amour perdu, mais plutôt celle d'un univers idéal inatteignable, fait de beauté et de sensualité, que le monde réel ne peut contenir. En ce sens, Fauré prend acte des représentations traditionnelles de l'Orient, mais amorce une prise de distance en le situant délibérément hors du monde, dans une perspective d'autoréférentialité. Sa démarche amorce une perspective positiviste analogue à celle qu'énoncent Denis et Taine, en ce sens que le but de sa mélodie est de " manifester quelque caractère essentiel et saillant plus complètement et plus clairement que ne le font les objets réels » (Taine [1882]1985, p. 373) ${ }^{8}$.

\section{« LAHORE » (1914) DE MAurice Delage}

Pourtant, la France colonialiste offre de multiples occasions de voyager à l'étranger. En 1889, dans le Harper's New Monthly Magazine, le peintre Benjamin-Constant publie un récit de voyage au $\mathrm{Maroc}^{9}$. Il y avait séjourné entre 1871 et 1873, puis en 1883. Dans son récit, Benjamin-Constant relate plusieurs détails de la vie de tous les jours, et consigne des éléments, des impressions ou des scènes susceptibles d'être mises en peinture. Ces notes lui seront utiles pour la production de plusieurs tableaux orientalisants, notamment La soif. Prisonniers marocains (1878; figure 5).

$6 \quad$ L'article est signé par les initiales A. B. Il se pourrait que ce soit le compositeur Albert Bertelin, qui écrivait parfois des chroniques dans La Revue musicale et qui a publié des souvenirs sur Fauré dans Musique et théâtre en 1925.

$7 \quad$ Voir le chapitre de Sarga Moussa, «Par delà l'Europe : musique, voix et sonorités dans Le Voyage en Orient de Lamartine », dans Bara et Ramaut 2012.

8 Taine applique cette phrase à l'art en général, mais ce n'est pas un contresens de l'appliquer plus spécifiquement à la mélodie de Fauré.

9 Ce récit a été traduit et publié dans le catalogue de l'exposition (Bondil 2014, p. 167-183). $\mathrm{Au} \mathrm{XIX}^{\mathrm{e}}$ siècle, les récits de voyage d'artistes sont nombreux, à la suite de celui de Lamartine (Voyage en Orient [1835], réédité par Sarga Moussa en 2000). En 1912, par exemple, Delage publie une «Lettre de 1'Inde» (Kandy, 4 mars) dans la Revue musicale S.I.M. 


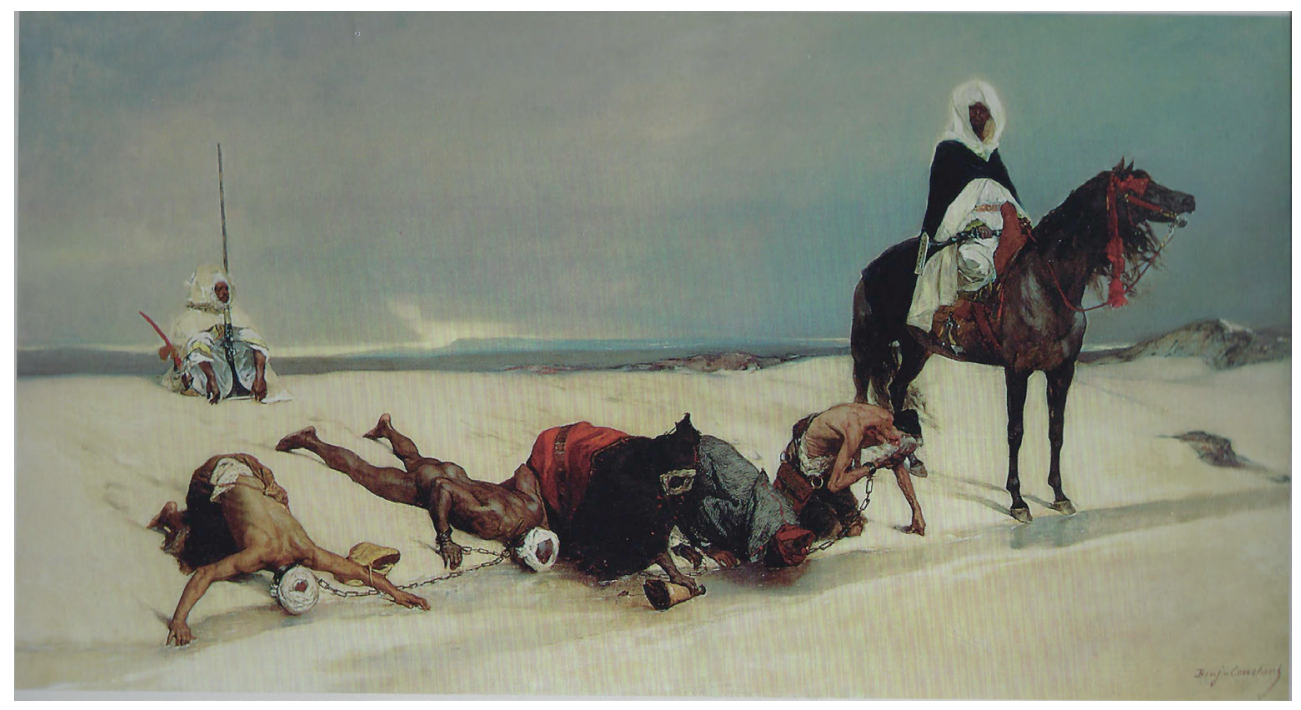

Figure 5 : Benjamin-Constant, La soif. Prisonniers marocains, 1878.

Huile sur toile, 117,2 213,4 cm. Signé b.d. : Benj-Constant. Londres, Christie's, 18 juin 1998, lot 3. Photo (C) Christie's Images Limited (1998).

Dans les années 1910, c'est le musicien Maurice Delage, dont la famille possédait des industries en Orient, qui fera un voyage au Japon et en Inde. Dans ces pays, il entend et il est touché par plusieurs musiques traditionnelles. Mais, contrairement aux compositeurs du $\mathrm{XIX}^{\mathrm{e}}$ siècle, Delage souhaite reproduire assez fidèlement les timbres, les échelles sonores, les textures et les rythmes des musiques indiennes. À la différence de Fauré, son Orient n'est pas fantasmé, il s'ancre dans la réalité sonore de l'Inde et s'écarte d'une représentation musicale de la sensualité féminine.

C'est entre 1912 et 1913 que Delage compose ses Quatrepoèmes hindous. L'œuvre a été créée lors du « concert mirifique et scandaleux » de la Société musicale indépendante, le 14 janvier 1914, sous les bons auspices de Ravel ${ }^{10}$. La seconde mélodie du cycle, "Lahore ", est révélatrice d'un orientalisme d'avant-garde. Au début, le violoncelle imite un instrument à cordes pincées de l'Inde du Nord, le sitar. Comme sur ce dernier, l'interprète pince les cordes en attaquant le son par dessous. Pour augmenter les possibilités de l'instrument, Delage demande de l'accorder différemment, en scordatura. L'effet est saisissant. À la fin de l'œuvre, la voix fait une longue vocalise sur le $a$, en solo, jouant avec les lèvres, qui s'ouvrent et qui se ferment à la manière $\mathrm{du}$ chant indien. Une imitation aussi proche des sonorités orientales est une attitude nouvelle en Occident. Delage ne force pas son modèle musical à entrer dans le cadre préexistant des préceptes de compositions qu'il a apprises à Paris. Au contraire, il crée une forme inédite à partir de ce qu'il a entendu en Inde et il renouvelle la technique instrumentale afin de produire des effets sonores non occidentaux.

Par contraste, dans le poème de Heinrich Heine choisi par Delage, l'Orient est représenté non plus avec réalisme, mais plutôt dans le registre de la métaphore. La

10 Pour le contexte d'avant-garde entourant ce concert, voir Duchesneau 1997, p. 90-91. 
scène se déroule dans un univers où le froid et la désolation servent de décor à un sapin isolé. On ne retrouve pas les projections fantasmagoriques habituelles de 1'Occident sur l'Orient. Le sujet poétique est tout bonnement un sapin et l'univers dont il rêve est uniquement végétal. Son correspondant oriental - un palmier - est lui aussi solitaire. L'endroit rêvé, c'est le sud, baigné de la chaleur bienfaisante.

La démarche compositionnelle de Delage n'est toutefois pas sans paradoxe. Deux des quatre poèmes du recueil sont de Bhartrihari, un poète indien d'expression sanskrite ayant vécu avant le viI ${ }^{\mathrm{e}}$ siècle. "Lahore », par contre, est de Heinrich Heine, un poète romantique allemand dont quelques vers avaient été mis en musique par Schubert dans son Chant du cygne. Du point de vue poétique, l'auditeur est toujours en présence d'un Orient fantasmé. De plus, malgré le fait que le titre des mélodies suggère un itinéraire de voyage réellement effectué, la musique entendue en Inde n'explique pas tout. Plusieurs thèmes ont été reproduits par Delage d'après un enregistrement sur disque, en $1905^{11}$, de musique traditionnelle de l'Inde. Cela relativise la question du voyage, fait sept ans plus tard. L'objet de l'œuvre composée par Delage est bien de la musique indienne, mais le contact fait sur place avec les pratiques musicales aura été préparé et remémoré par un enregistrement sonore.

L'idée d'employer des figures musicales préexistantes pour évoquer l'Orient n'est pas nouvelle. Du Désert (1844) de Félicien David à Thaïs (1894) de Massenet, ces figures sont réutilisées par les compositeurs et reconnues par le public. On peut se demander alors ce qui distingue l'orientalisme de Delage de celui de Félicien David, qui avait lui aussi noté des musiques locales lors de son séjour en Afrique en $1835^{12}$. L'avènement de l'enregistrement sonore avec le phonographe, au tournant du siècle, modifie l'horizon d'attente et entraîne un renouvellement des traits syntaxiques exotiques, qui commencent à être davantage fondés sur la réalité d'un ailleurs. On commence ainsi à disposer pour la musique de l'équivalent de ce que les peintres avaient rapporté lors de leurs voyages, en reproduisant des objets ou des lieux exotiques avec un souci d'exactitude. D'autre part, même si elles deviennent réelles, les figures exotiques s'inscrivent dans la démarche d'avant-garde du compositeur. Certes, Delage ne veut pas faire une reproduction phonographique de la musique indienne - celle-ci existe déjà. À partir de "thèmes » exotiques, Delage produit une œuvre qui fait évoluer son propre langage, qui trace une voie à la modernité par sa forme et par son instrumentation. Il cherche à produire une sorte d'équivalent du Pierrot lunaire de Schönberg, que lui et son entourage considèrent comme un modèle à suivre ${ }^{13}$.

11 Voir l'article de Jann Pasler, « Maurice Delage », dans le Grove Music Online.

12 Voir Defrance 1994, en ligne.

13 Voir Duchesneau 1997, p. 212. 


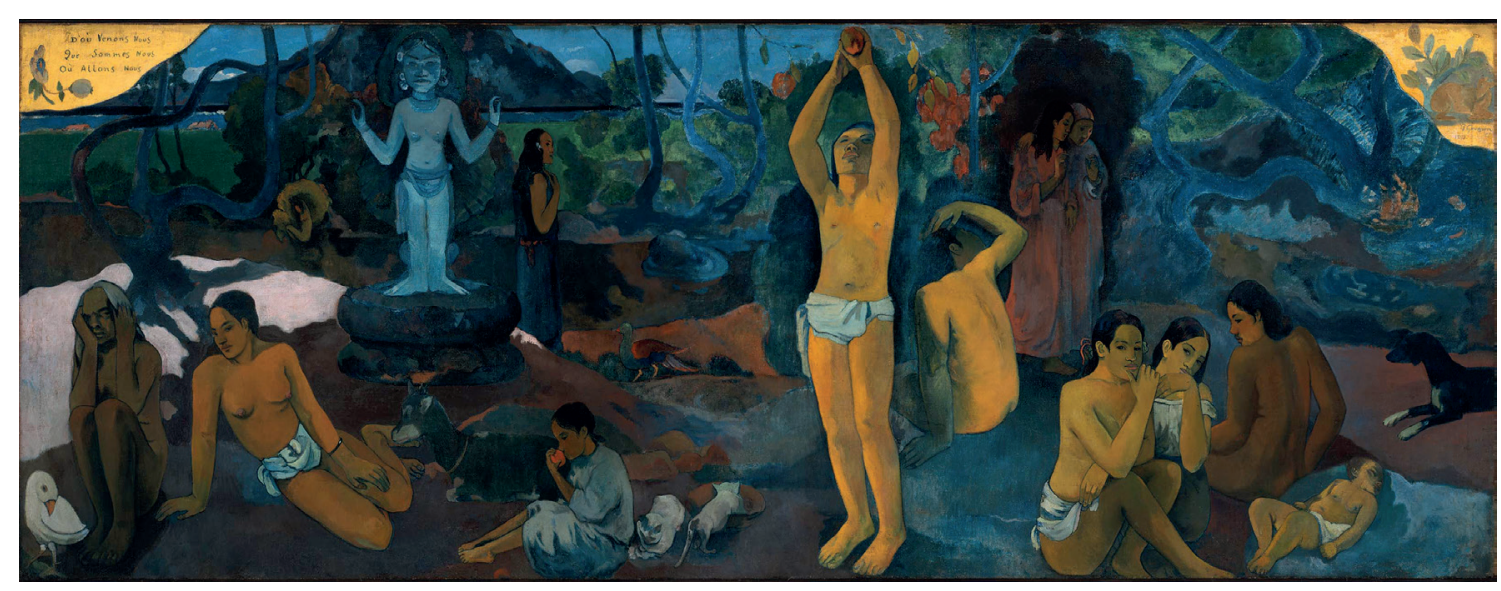

Figure 6: Paul Gauguin, D'où venons-nous ? Que sommes-nous ? Où allons-nous ?, 1897-1898. Huile sur toile, 139, 1 x 374,6 cm. Boston, Musée des beaux-arts de Boston. Source: Museum of Fine Arts Boston, sous licence Domaine public via Wikimedia Commons.

Pour expliquer le passage de l'orientalisme du XIX ${ }^{e}$ siècle à celui du Xx ${ }^{e}$ siècle, l'article intitulé « Race, Orientalism, and Distinction » de Jann Pasler (2000), jette un éclairage intéressant. L'auteure remarque avec pertinence le fossé qui sépare Albert Roussel de Maurice Delage. Alors que Roussel collectionne des thèmes pour produire une œuvre « orientaliste à la française », Delage prend la musique indienne pour ce qu'elle est en elle-même et non pour l'asservir à sa propre culture. Le voyage en Orient, bien que réel, n'est pas pour Delage un simple exercice de collection de thèmes entretenant des clichés préexistants, mais plutôt une démarche de réelle ouverture à une musique différente de celle qui se fait en Occident. C'est une authentique démarche d'altérité. Sans doute que son présupposé est aussi, comme cela l'a été à un certain moment pour Gauguin, que les civilisations traditionnelles ont conservé des modes d'expression en quelque sorte non souillés par la civilisation occidentale et que les connaître permet de retrouver une pureté expressive qui avait été perdue (Mackintosh 2007) ${ }^{14}$. Retrouver cette source pure de l'expression se conjugue parfaitement avec la recherche de l'avantgarde de l'époque, qui n'a pas pour but de produire la nouveauté pour elle-même, mais de retrouver une vérité dans l'expression, en la libérant de l'académisme et de conventions devenues stériles.

\section{« Nahandove » (1926) De Maurice Ravel}

Tout en poursuivant un même but - celui d'actualiser la musique de son temps au moyen d'éléments exotiques -, Ravel emprunte un chemin très différent. Il y a chez lui ce que Barbara Kelly (2000, p. 10), mais également Robert Orledge (2000), qualifie d'orientalisme de distanciation (Kelly 2000, p. 39-40). Ravel considérait la sincérité « comme le plus grave défaut en art, parce qu'elle exclut la possibilité de choix [...]

14 Concernant l'attrait de l'exotisme pour Gaugin, voir plus particulièrement Mackintosh 2007, p. 71-72. 
La chose la plus fascinante en art est de tenter de surmonter les difficultés » (cité dans Kaminsky 2000, p. $186^{15}$ ). Ravel refuse donc l'orientalisme en tant qu'imitation, mais l'assume pleinement dans son potentiel d'accroissement de la palette imaginative du musicien. Désormais, le passé ou les contrées lointaines ne sont plus considérés comme nostalgie d'un idéal, mais comme actualisation d'un héritage, comme un potentiel de créativité riche pour la modernité. L'art musical est renvoyé à lui-même, mais dans un jeu d'intertemporalité.

Le choix de poèmes que fait Ravel contribue à renforcer cette esthétique moderne qui fait écho au passé. L'auteur du recueil poétique Chansons madécasses, Évariste de Parny, est né à l'île de La Réunion - une colonie française de l'océan Indien au XVIII ${ }^{\mathrm{e}}$ siècle. Il prétend avoir traduit en français un ancien recueil provenant de Madagascar, madécasse signifiant malgache. Mais on ne connaît pas l'original et, dans l'histoire de la littérature, les faux ne manquent pas. Et même si ce n'était pas un faux, comment pourrait-on exclure l'hypothèse que Parny n'aurait pas teinté les poèmes de ses propres opinions, ne serait-ce que par sa traduction ? On sait par ses lettres que Parny était un anticolonialiste opposé à l'esclavage : le propos du poème n'en est que plus suspect. De plus, on retrouve dans le poème des accents dramatiques, voire érotiques, qui n'ont rien à voir avec la "vérité exotique ". On retrouvait cet érotisme dans «Asie» (Shéhérazade) de Ravel, mais il était soutenu par un grand orchestre. C'était une sensibilité toute dix-neuviémiste, où l'exotisme servait de véhicule à l'érotisme. Dans " Nahandove ", presque à l'encontre du poème, Ravel imagine une expression de l'érotisme plus retenue et intime que celle véhiculée par les clichés de l'orientalisme français, car la musique possède le pouvoir d'orienter l'expression d'un poème. Par le refus de l'érotisme exotique du XIX ${ }^{\mathrm{e}}$ siècle et par ses préoccupations anticolonialistes, le compositeur s'écarte de l'orientalisme érotisant tel que représenté par des peintres comme Benjamin-Constant (figure 7), et rejoint les idéaux de Gauguin (figure 6), qui désérotise les femmes nues et replace les corps dans leur environnement naturel, dans leur pureté primitive.

Dans les Chansons madécasses, Ravel, qui est un grand orchestrateur, choisit délibérément de faire appel à des moyens restreints : petit ensemble instrumental (voix, flûte, violoncelle et piano, qui n'est pas sans rappeler Schönberg), matériel mélodique réduit et répétitif, contrepoint où les voix ne se mêlent pas. L'individualité timbrale et mélodique des lignes vocales et instrumentales remplace la sensation de masse, le fondu d'un grand orchestre. Il y a presque une sorte d'austérité dans cette œuvre, tant son caractère est minimaliste. Certes, à la troisième strophe, Ravel introduit au piano un rythme plus haletant, une montée de la tension. Mais il s'agit plutôt d'une émotion venant de l'attente que d'une tension érotique. Lorsque les amants se rencontrent et entrent en contact physiquement, à la cinquième strophe, il y a certes une certaine animation, mais elle demeure contenue, plus près du registre de la volupté que des caresses qui brûlent tous les sens.

15 Traduction de : "I consider sincerity to be the greatest defect in art, because it excludes the possibility of choice. Art is meant to correct nature's imperfections... The most interresting thing in art is to try to overcome difficulties. " Kaminsky cite lui-même une entrevue faite par André Révész, " The great musician Maurice Ravel talks about his art ", dans A.B.C. de Madrid, $1^{\text {er }}$ mai 1924, p. $431-435$ (p. 433 pour la citation). 
Dans une conférence qu'il a donnée au Rice Institute (Houston) en 1928, Ravel explique comment se joue l'équilibre entre des éléments culturels exotiques et sa propre individualité, comme compositeur. Pour lui, nationalisme, exotisme et individualité sont en constante négociation au sein du processus créateur. Il explique que le blues est l'un des genres les plus américains qui soit, même si le blues vient d'Afrique. Des musiciens lui ont demandé pourquoi il avait fait un emprunt au blues dans le second mouvement de sa sonate pour violon et piano. Ravel affirme que cette sonate est réellement française et ravélienne. Le même blues, traité par un autre musicien, français ou non, n'aurait pas été traité de la même manière que par lui. L'individualité d'un compositeur est plus forte que le matériel qu'il peut éventuellement s'approprier (Pilarski 1964). Dans la même conférence, Ravel ajoute que Schönberg l'a influencé, non par son système, mais plutôt par son esthétique : "I am quite conscious of the fact that my Chansons Madécasses are in no way Schönbergian, but I do not know whether I ever should have been able to write them had Schönberg never written " (cité dans ibid., p. 218).

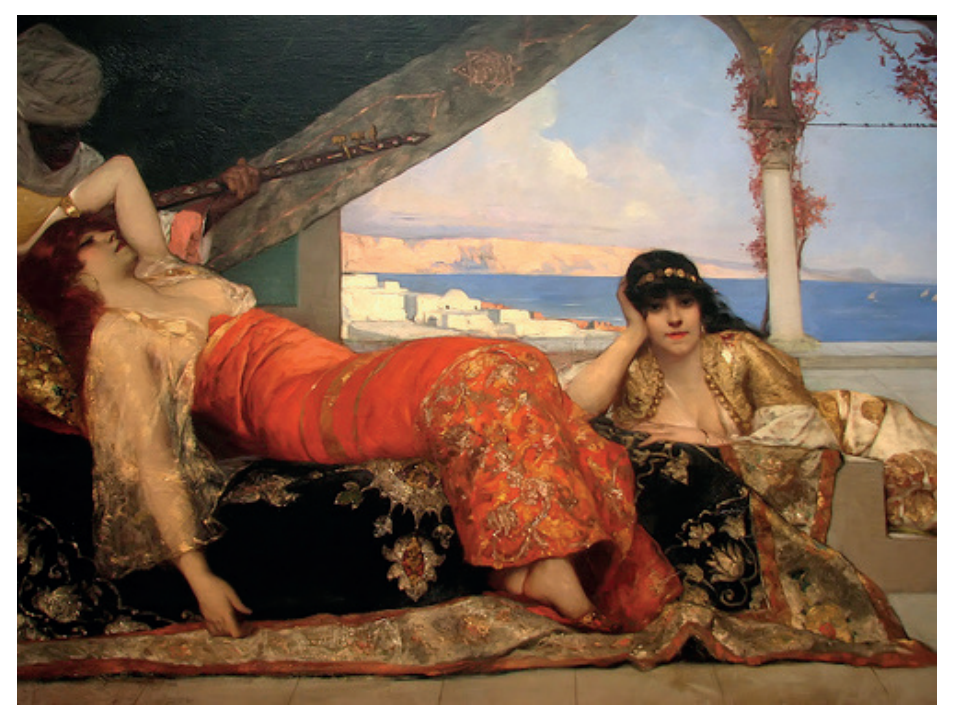

Figure 7 : Benjamin-Constant, La favorite de l'émir, vers 1879.

Huile sur toile, 142,2 $221 \mathrm{~cm}$. Signé b.g. : Benj-Constant. Washington, National Gallery of Art.

Courtesy of the United States Naval Academy Museum. 2010.95.1.

Sous certains aspects, la représentation ravélienne de l'Orient offre des points communs avec celle de Delage, par la nouveauté du matériel musical, le traitement inédit des timbres et le décentrement culturel qu'il suscite. Toutefois, Ravel n'a pas fait de voyage à Madagascar. Il s'est plutôt fondé sur l'idée que l'on se faisait, en Europe, de la musique malgache (James 1990). Le véritable « voyage » de Ravel nous emporte plutôt au pays de l'avant-garde. Notamment, son souci d'organiser la forme de manière minimaliste s'inscrit dans le néoclassicisme français de l'entre-deux-guerres. Tout comme Delage, Ravel souhaite présenter les cultures traditionnelles de telle sorte que leur pureté artistique, réelle ou imaginée, devient un modèle pour l'Occident. Les rôles sont maintenant inversés : ce n'est plus l'Occident tout-puissant qui civilise les « peuples primitifs », mais l'art primitif qui apporte au musicien occidental une 
fraîcheur d'inspiration. C'est un Orient imaginé, certes, mais dépouillé de son aura merveilleuse et sensuelle, et surtout intégré dans une démarche où prévalent 1'originalité et la nouveauté du langage recherchées par le compositeur.

\section{« LA Flûte de Pan » (1899) de Claude Debussy}

Avec Debussy, nous entrons de plain-pied dans un Orient imaginaire où la réalité objective est sciemment reléguée au rôle de prétexte. C'est un Orient évoqué, mais non nommé, puisque dans une perspective symboliste, nommer ${ }^{16}$ l'objet serait supprimer une large part de la jouissance du poème. Le véritable sujet d'une œuvre symboliste n'est pas l'objet, le sentiment ou la situation évoquée, mais l'effet produit par ceux-ci. La musique cherche à recréer le même type d'expérience que pourraient procurer ces objets. Par exemple, dans "La flûte de Pan », Debussy cherche à faire vivre chez l'auditeur les mêmes sensations ou sentiments que ceux provoqués par les lèvres des amoureux qui se posent l'une sur l'autre, mais sans les nommer. La flûte agit comme substitut à la situation, comme symbole. Dans les Trois chansons de Bilitis, c'est la Grèce antique qui est identifiée comme Orient. Le recueil de Pierre Louÿs, où Debussy puise les poèmes, est un "vrai faux ", en ce sens que Pierre Louÿs joue sur l'hellénisme ambiant ${ }^{17}$ pour publier un recueil qu'il fait d'abord passer comme la traduction d'un manuscrit grec retrouvé. La supercherie atteint des proportions appréciables puisque le recueil s'ouvre par une "Vie de Bilitis » qui ressemble à une véritable biographie ${ }^{18}$. Si l'astuce a semblé vraisemblable, c'est non seulement parce que Louÿs est un excellent connaisseur du grec ancien, mais aussi parce qu'il s'inscrit dans une sensibilité du temps qui allie la Grèce ancienne avec l'orientalisme ${ }^{19}$.

16 "Nommer un objet, c'est supprimer les trois quarts de la jouissance du poème qui est faite du bonheur de deviner peu à peu : le suggérer, voilà le rêve » (Huret 1891, p. 60).

17 À ce sujet, voir l'article de Christophe Corbier (2010), notamment, lorsqu'il écrit : « Dans le dernier quart du Xix ${ }^{e}$ siècle, depuis les travaux de Gevaert (Histoire et théorie de la musique de l'Antiquité, 1875-1881) jusqu'à la découverte du fragment musical de l'Oreste d'Euripide en 1890 par Carl Wessely et la transcription des deux hymnes delphiques en 1893-1894 par Théodore Reinach et Henri Weill, qui suscitent à la fois surprise et enthousiasme dans le public, l'art musical des anciens Grecs se pare de couleurs inconnues et étranges. En lisant les écrits des musicologues et des hellénistes, en écoutant les ouvres antiques, chacun peut percevoir la part de mystère et de rêve que conserve la Grèce ancienne " (Corbier 2010, p. 328). Nous pouvons ajouter que Fauré a harmonisé un hymne delphique en 1894 et qu'il a composé son opéra Pénélope entre 1907 et 1912.

18 Certains critiques de l'époque, comme Paul Ginistry, étaient toutefois demeurés dubitatifs : "Si c'est un jeu littéraire, il est charmant. Si c'est une traduction véritable, ce doit être une traduction assez libre, car, tant que s'évoque par là l'esprit grec, les poèmes paraissent imprégnés aussi quelque peu de l'esprit moderne " (Gil Blas, 5 janvier 1895, cité par Clive 1978, p. 111).

19 Il faut aussi ajouter que par cette publication érotico-hellénique, Louÿs cherche probablement un succès qui lui procurera des revenus lui permettant de vivre décemment (voir Dumont 1985, p. 143-150). En outre, dans une perspective orientaliste, il est intéressant de noter que les Chansons de Bilitis ont été écrites en grande partie à Constantine (Algérie) en 1894 et qu'elles ont probablement été inspirées par la maîtresse mauresque de Louÿs, Meryem ben Ali. 
Au début de "La flûte de Pan», Debussy donne à sa musique une carrure rythmique grecque, avec le mètre iambique brève-longue. L'introduction du piano place bien ce rythme, qui procure un effet antiquisant. Ici, nous ne pouvons pas parler d'une figure musicale exotique, mais plutôt d'une idée musicale qui renvoie au poème. Nous sommes dans l'autoréférentialité poétique, dans le sens où l'image musicale ne renvoie plus à un paysage ou à une scène pouvant se passer réellement en Orient, mais plutôt à une pratique artistique, celle du poème déclamé. Étant transféré du poétique au musical, le mètre agit comme une figure. Les sonorités modales renforcent cette manière moderne d'évoquer ce qui est ancien.

Par ailleurs, la question de la représentation de la femme, sous certains aspects, se joue dans le registre traditionnel de l'érotisme. Mais la langue de Louÿs est tellement codée, stylisée, que la séduction est davantage un effet esthétique qu'une réalité sensuelle ${ }^{20}$. D'ailleurs, la musique de Debussy précipite la sortie hors des codes traditionnels de l'exotisme et tourne l'attention de l'auditeur vers la musique elle-même. On entend le cri des grenouilles, le temps qui passe, le son de la flûte : ce sont des éléments anecdotiques par rapport au sujet poétique.

\section{UN PARCOURS VERS LA SENSORIALITÉ DE L'ART}

À partir de la citation de Denis énoncée au début de cet article, nous avons expliqué comment l'imaginaire oriental peut être déclenché par la matérialité de l'œuvre. En peinture, la conduite du regard s'amorce par une surface plane recouverte de couleurs en un certain ordre assemblées. En musique, la conduite d'écoute est guidée par des sons en un certain ordre assemblés. La musique n'évoque plus un paysage ou des sensations en les imitant, mais plutôt en cherchant à faire vivre chez l'auditeur des sensations comparables à celles vécues par le sujet poétique, le " je " du poème.

En détachant la mélodie de son sujet narratif, Debussy ouvre la voie à la prédominance de l'expression par la forme et à des stratégies de création orientées vers l'imaginaire de celui qui écoute. Dans cette foulée, l'orientalisme d'avantgarde $^{21}$ s'inscrira globalement dans deux catégories différentes : une première où l'orientalisme se réduit à une trace, comme une impression fugitive qui déclenche l'imaginaire, et une seconde où l'orientalisme est assimilé à une démarche axée sur la forme. Denis écrivait que l'art « est une création de notre esprit dont la nature [ou même l'orientalisme] n'est que l'occasion »(Denis 1993, p. 162). C'est dans cette perspective que l'Orient debussyste évolue vers une trace et qu'il n'est plus l'objet principal de l'expression. Dans «Le Faune »(1904), tiré du second volume des

20 Comme en témoignent les nombreux écrits érotiques de Louÿs, ainsi que certains autres poèmes du recueil de Bilitis - notamment «L'arbre »-, l'auteur se sert de la Grèce antique et de son esthétique pour styliser un projet finalement très libertin.

21 Par orientalisme d'avant-garde, nous entendons une tendance des compositeurs du $\mathrm{Xx}^{\mathrm{e}}$ siècle à tirer prétexte des représentations musicales de l'Orient pour servir une démarche compositionnelle originale où le langage musical se veut inédit et détaché des significations d'origine. 
Fêtes galantes, Debussy adopte un caractère humoristique, voire satyrique. L'Orient, dépouillé de son aura mirifique, devient l'objet de moquerie. Il est réduit à n'être plus qu'un Faune de terre cuite, qui regarde la mélancolie de jadis comme une expression dépassée remplacée par la fête. Sa mise à distance est double : il est dépouillé de ses connotations sensuelles et le motif musical orientalisant dans le registre grave du piano - que l'on peut rapprocher du son du tambourin et de la danse - est réduit à sa plus simple expression, à un motif inlassablement répété.

Nous pouvons étendre les conséquences de cette approche à l'extérieur du domaine de la mélodie, en dehors de toute référentialité à un texte. Tirée des Estampes (1903) pour piano, "La Soirée dans Grenade " appartient à la catégorie de trace ${ }^{22}$. Le titre - qui est d'ailleurs placé à la fin, comme simple suggestion - demeure l'un des rares moyens qu'utilise Debussy pour évoquer l'exotisme. Certes, le rythme de la habanera, au début de l'œuvre, évoque clairement l'Orient, mais il est ensuite tellement décontextualisé qu'il devient un simple déclencheur de sensations. Debussy pousse ici l'avant-garde à une conséquence assez radicale, en sortant du cadre étroit de la représentation figurative pour ouvrir le motif à un réseau de significations beaucoup plus ouvert. Pour reprendre la citation de Bartoli par la négative, on pourrait dire que la habanera devient un paramètre isolé qui ne parvient plus à s'imposer dans un réseau de figures exotisantes (Bartoli 2000, p. 65). Le titre du recueil est d'ailleurs indicateur de cette attitude créatrice : l'estampe est une gravure, une image imprimée sur papier à partir d'une planche de bois ou d'une plaque métallique. L'estampe est la trace laissée par un modèle. Pour Debussy, la partition est conçue comme cette plaque, alors que le son s'imprime chez l'auditeur. L'œuvre, c'est finalement la trace laissée par le son de celui qui joue en celui qui écoute.

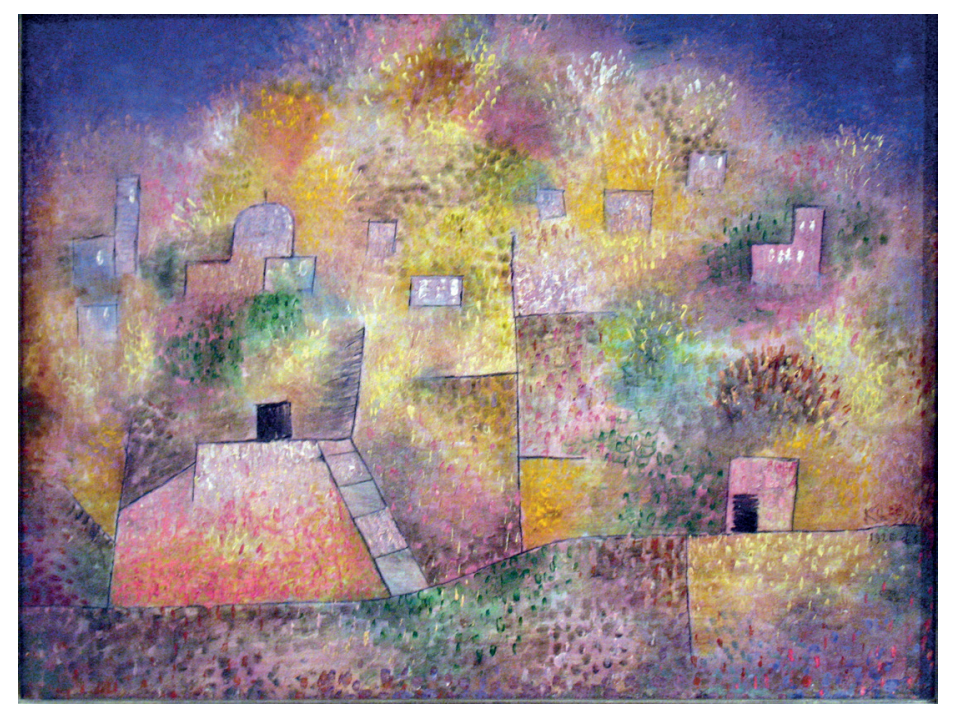

Figure 8: Paul Klee, Jardin d'agrément oriental, 1925. Huile sur carton, $42,3 \times 51 \mathrm{~cm}$. New York, The Metropolitain Museum of Art. Source : anagoria, sous licence Domaine public via Wikimedia Commons.

22 Il faut se rappeler que dans l'univers de Benjamin-Constant, Grenade et l'Espagne mauresque font partie de l'Orient au sens large. Voir Bondil 2014, p. 21. 
Une seconde catégorie d'œuvres concerne l'utilisation de motifs orientaux, considérés comme un outil de renouvellement de l'expression, dans une démarche qui met l'accent sur la forme. La recherche d'une musique qui est d'abord matière sonore et structuration de sons a inspiré un peintre comme Paul Klee. Klee est fasciné par le pouvoir d'expression des structures musicales. Dans le Jardin d'agrément oriental (figure 8), on reconnaît certaines formes orientalisantes, mais l'expression de l'œuvre est tournée vers leur organisation géométrique, vers l'expression par la disposition des formes et par l'interaction entre les couleurs. Est-ce à dire que la musique aurait préséance sur la peinture dans l'orientalisme d'avant-garde? Assurément, non. Dans un jeu de va-et-vient constant, peintres et musiciens s'inspirent mutuellement. Dans Le pays fertile de Paul Klee, Pierre Boulez explique en quoi la pensée des structures de Klee l'a inspiré dans sa démarche de compositeur. Nous faisons nôtre la dernière phrase de son livre puisqu'elle résume l'idée conductrice de cet article : "si la structure force l'imagination à entrer dans une nouvelle poétique, alors, on est, en effet, en pays fertile» (Boulez 1989, p. 175).

Lorsqu'il a publié son aphorisme, Denis n'avait sûrement pas imaginé à quel point la musique et la peinture $\mathrm{du} \mathrm{Xx}^{\mathrm{e}}$ siècle s'orienteraient vers des conséquences radicales quant à l'autonomie des lois de l'art par rapport à la représentation de la nature ou d'un récit. Son énoncé positiviste présupposait la préséance des lois propres au médium, mais non la disparition d'une référentialité au sujet de l'œuvre. A posteriori, les développements de l'art abstrait et de la recherche du son en lui-même dans la musique donnent une dimension prophétique à ses réflexions. L'atténuation, voire la disparition du lien narratif a permis que les objets orientalisants - qu'ils soient picturaux ou musicaux - puissent devenir les véhicules d'une vision artistique renouvelée, où l'expression se concentre désormais sur les lis propres au médium. L'Orient ne sert plus à évoquer le merveilleux. Abstrait de son contexte d'origine, il séduit par ses potentialités de renouvellement du langage musical occidental, devenant ainsi un matériel de choix pour les compositeurs d'avant-garde.

BIBLIOGRAPHIE

A. B. (1906), "Les œuvres récemment exécutées. Les Roses d'Ispahan, de G. Fauré (Concerts Sechiari) ", La Revue musicale, 6 ${ }^{\mathrm{e}}$ année, $\mathrm{n}^{\circ} 24$ (15 décembre), p. 575-576. Disponible en ligne : http://bluemountain.princeton.edu/bluemtn/cgi-bin/bluemtn?a=d\&d=bmtnabj1906121501\&e=-------en-20--1--txt-txIN------, consulté le 7 décembre 2015.

Barbe, Michèle (2011), Musique et arts plastiques. La traduction d'un art par l'autre, Paris, L'Harmattan.

Bara, Olivier, et Alban Ramaut (2012), Généalogies du romantisme musical français, Paris, Vrin.

Bartoli, Jean-Pierre (2000), "Propositions pour une définition de l'exotisme musical et pour une application en musique de la notion d'isotopie sémantique ", Musurgia, vol. 7, $\mathrm{n}^{\circ}$ 2, p. 61-71. Disponible en ligne : http://www.jstor.org/stable/40591372?seq=1, consulté le 7 décembre 2015.

Bondil, Nathalie (dir.) (2014), Benjamin-Constant. Merveilles et mirages de l'orientalisme, catalogue d'exposition, Toulouse, Musée des Augustins, Montréal, Musée des beaux-arts de Montréal, Paris, Hazan.

Bouillon, Jean-Paul (2006), Maurice Denis. Six essais, Parais, Somogy. 
Boulez, Pierre (1989), Le pays fertile. Paul Klee, Paris, Gallimard.

Clive, Harry P. (1978), Pierre Loü̈s (1870-1925). A Biography, Oxford, Oxford University Press.

Corbier, Christophe (2010), "La Grèce de Charles Koechlin », dans Philippe Cathé, Sylvie Douche et Michel Duchesneau (dir.), Charles Koechlin compositeur et humaniste, Paris, Vrin, p. 327-347.

Debussy, Claude (1888), Ariettes oubliées, Paris, Veuve Girod. Disponible sur http://imslp.org.

Debussy, Claude (1899), Trois chansons de Bilitis, Paris, Fromont. Disponible sur http://imslp.org.

Defrance, Yves (1994), "Exotisme et esthétique musicale en France. Approche socio-historique ", Cahiers d'ethnomusicologie, vol. 7, p. 191-210. Disponible en ligne : http://ethnomusicologie.revues. org/1409, consulté le 7 décembre 2015.

Delage, Maurice (1912), «Lettre de 1'Inde » (Kandy, 4 mars 1912), Revue musicale S.I.M., $2^{\mathrm{e}}$ année, $\mathrm{n}^{\circ} 6$ (15 juin), p. 72-74. Disponible en ligne : http://bluemountain.princeton.edu/bluemtn/cgi-bin/ bluemtn? $\mathrm{a}=\mathrm{d} \& \mathrm{~d}=\mathrm{bmtnabh} 19120615-01 \& \mathrm{e}=---\mathrm{-}_{--e n-20-1-\text {-txt-txIN------, }}$ consulté le 7 décembre 2015.

Delage, Maurice (1914), Quatre poèmes hindous, Paris, Durand.

Denis, Maurice (1914), Théories (1890-1910). Du symbolisme de Gauguin vers un nouvel ordre classique, Paris, Rouart.

Denis, Maurice (1993), Le ciel et l'Arcadie, textes réunis, présentés et annotés par Jean-Paul Bouillon, Paris, Hermann.

Duchesneau, Michel (1997), L'avant-garde musicale et ses sociétés à Paris de 1871 à 1939, Liège, Mardaga.

Dumont, Paul-Ursin (1985), Pierre Loü̈s, l'ermite du hameau, Vendôme, Libraidisque.

Fauré, Gabriel (1885), Quatre mélodies, op. 39, Paris, Hamelle. Disponible sur http://imslp.org.

Gombrich, Ernst Hans (2001), Histoire de l'art, Paris, Phaidon.

Heine, Heinrich (1827), Buch der Lieder, Hambourg, Hoffmann und Campe. Disponible en ligne : http://www.staff.uni-mainz.de/pommeren/Gedichte/BdL, consulté le 7 décembre 2015.

Huret, Jules (1891), « M. Stéphane Mallarmé », dans Enquête sur l'évolution littéraire, Paris, Bibliothèque Charpentier. Disponible en ligne : https://www.uni-due.de/lyriktheorie/texte/1891 huret.html, consulté le 7 décembre 2015.

James, Richard S. (1990), « Ravel's "Chansons madécasses." Ethnic Fantasy or Ethnic Borrowing ? , The Musical Quaterly, vol. 74 n $^{\circ}$ 3, p. 360-384.

Junod, Philippe (2006), Contrepoints. Dialogues entre musique et peinture, Genève, Éditions Contrechamps.

Kaminsky, Peter (2000), "Vocal Music and the Lures of Exoticism and Irony », dans Deborah Mawer (dir.), Cambridge Companion to Ravel, Cambridge, Cambridge University Press, p. 162-187.

Kelly, Barbara (2000), "History and Hommage », dans Deborah Mawer (dir.), Cambridge Companion to Ravel, Cambridge, Cambridge University Press, p. 7-26.

Lamartine, Alphonse de ([1835]2000), Voyage en Orient, réédité par Sarga Moussa, Paris, H. Champion. Édition de 1913 disponible en ligne : http://gallica.bnf.fr/ark:/12148/bpt6k3735995, consulté le 7 décembre 2015.

Leconte de Lisle (1884), Poèmes tragiques, Paris, Alphonse Lemerre. Disponible en ligne : http://gallica.bnf.fr/ark:/12148/btv1b8618394d.r=leconte de lisle poèmes tragiques, consulté le 7 décembre 2015.

Le Grand Robert (2009), CD-Rom, $2^{e}$ édition. Article « positivisme ».

Locke, Ralph P. (2007), «A Broader View of Musical Exoticism», The Journal of Musicology, vol. 24 n 4 , p. 477-521, http://www.esm.rochester.edu/uploads/"A-Broader-View-of-Musical-Exoticism". pdf, consulté le 7 décembre 2015. 
Locke, Ralph P. (2009), Musical Exoticism. Images and Reflections, Cambridge, Cambridge University Press.

Louÿs, Pierre (1894), Les Chansons de Bilitis, Paris, Société du Mercure de France.

Mackintosh, Alistair (2007), «Symbolisme et art nouveau », dans David Britt (dir.), L'art moderne, de l'impressionnisme au post-modernisme, Paris, Thames \& Hudson, p. 59-108.

Moussa, Sarga (2012), " Par delà l'Europe. Musique, voix et sonorités dans Le Voyage en Orient de Lamartine ", dans Olivier Bara et Alban Ramaut (dir.), Généalogies du romantisme musical français, Paris, Vrin.

Nattiez, Jean-Jacques (2008), Lévi-Strauss musicien. Essai sur la tentation homologique, Arles, Actes Sud.

Nattiez, Jean-Jacques (2010), La musique, les images et les mots. Du bon et du moins bon usage des métaphores dans l'esthétique comparée, Montréal, Fides.

Orledge, Robert (2000), « Evocations of Exoticism », dans Deborah Mawer (dir.), Cambridge Companion to Ravel, Cambridge, Cambridge University Press, p. 27-46.

Parny, Évariste de (1787), Chansons madécasses traduites en français, suivies de poésies fugitives, Londres/ Paris, Hardouin et Gattey. Disponible en ligne : http://gallica.bnf.fr/ark:/12148/bpt6k1088229. $\underline{\mathrm{r}=\text { chansons madécasses, }}$ consulté le 7 décembre 2015.

Pasler, Jann, « Delage, Maurice », Grove Music Online. Oxford Music Online, Oxford University Press, consulté le 31 mars 2015.

Pasler, Jann (2000), «Race, Orientalism, and Distinction », dans Georgina Born et David Hesmondhalg (dir.), Western Music and its Others. Difference, Representation, and Appropriation in Music, Berkeley, University of California Press, p. 86-118.

Pilarski, Bohdan (1964), «Une conférence de Maurice Ravel à Houston (1928) », Revue de Musicologie, vol. $50, \mathrm{n}^{\circ} 129$, p. 208-221.

Ravel, Maurice (1926), Chansons madécasses, Paris, Durand. Disponible sur http://imslp.org.

Roque, Georges (2003), Qu'est-ce que l'art abstrait ? Une histoire de l'abstraction en peinture, Paris, Gallimard.

Taine, Hippolyte ([1882]1985), Philosophie de l'art, Paris, Fayard. Plusieurs éditions disponibles sur http://gallica.bnf.fr.

Verlaine, Paul (1869), Fêtes galantes, Paris, Alphonse Lemerre. Disponible en ligne : http://gallica.bnf.fr/ark:/12148/btv1b86268407.r=verlaine fêtes galantes, consulté le 7 décembre 2015. 


\section{ANNEXE : Les poèmes originaux des mélodies et leur appropriation par le compositeur.}

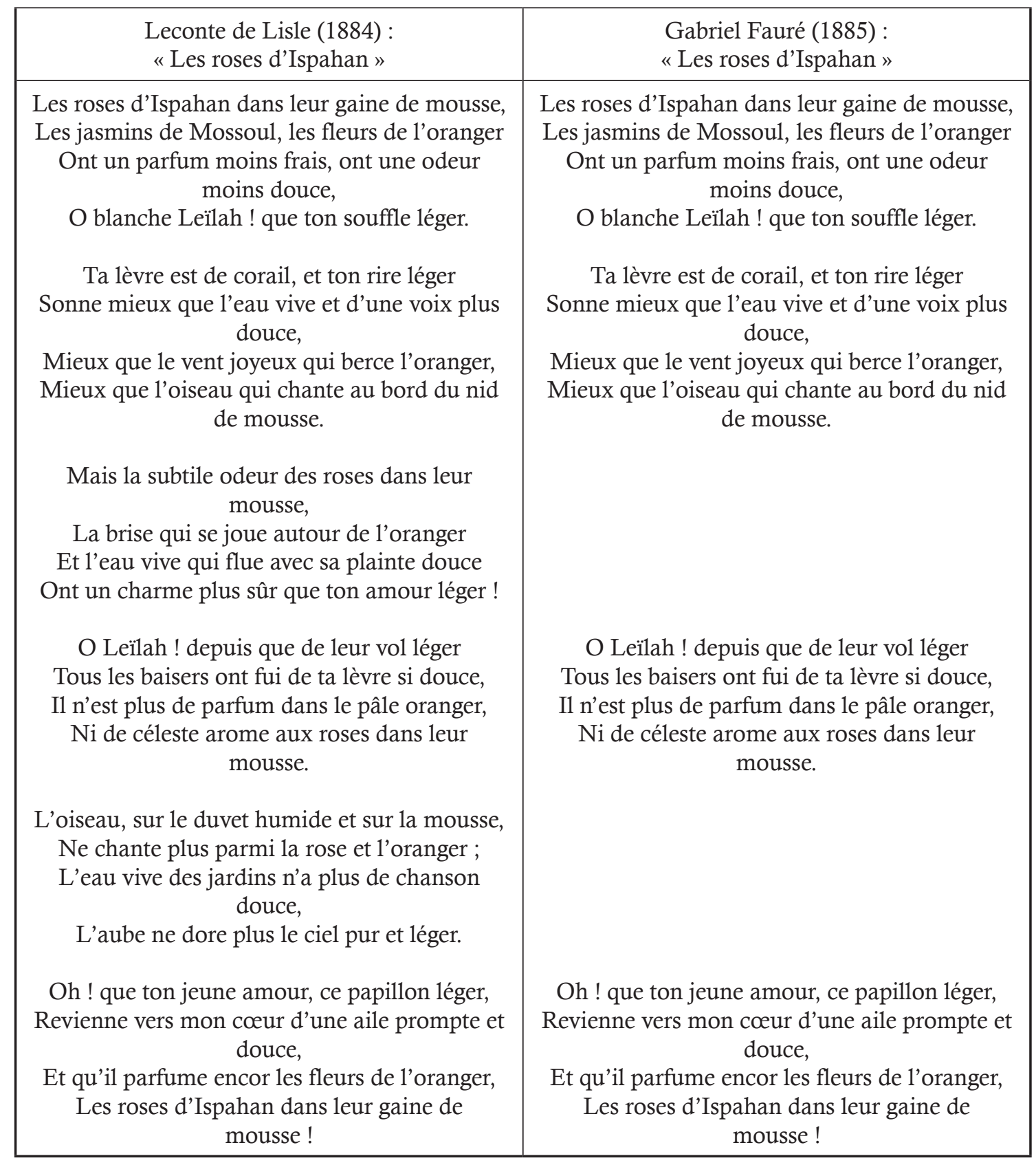




\begin{tabular}{|c|c|}
\hline Heinrich Heine (1827) : & Maurice Delage (1914) : \\
«Ein Fichtenbaum steht einsam » & "Lahore » \\
\hline Ein Fichtenbaum steht einsam & Un sapin isolé se dresse sur une montagne \\
Im Norden auf kahler Höh'. & aride du nord. \\
Ihn schläfert; mit weißer Decke & Il sommeille. \\
Umhüllen ihn Eis und Schnee. & $\begin{array}{c}\text { La glace et la neige l'environnent d'un manteau } \\
\text { blanc. }\end{array}$ \\
& Il rêve d'un palmier qui, là-bas, dans l'Orient \\
Er träumt von einer Palme, & lointain, \\
Die, fern im Morgenland, & se désole, solitaire et taciturne, \\
Einsam und schweigend trauert & sur la pente de son rocher brûlant. \\
Auf brennender Felsenwand. & \\
\hline &
\end{tabular}

Évariste de Parny (1787) et Maurice Ravel (1926) : « Nahandove»

Nahandove, ô belle Nahandove!

L'oiseau nocturne a commencé ses cris,

la pleine lune brille sur ma tête, et la rosée naissante humecte mes cheveux.

Voici l'heure: qui peut t'arrêter,

Nahandove, ô belle Nahandove!

Le lit de feuilles est préparé ; je l'ai parsemé de fleurs et d'herbes odoriférantes ; il est digne de tes charmes.

Nahandove, ô belle Nahandove?

Elle vient. J'ai reconnu la respiration précipitée que donne une marche rapide ;

j'entends le froissement de la pagne qui l'enveloppe ;

c'est elle, c'est Nahandove, la belle Nahandove !
Reprends haleine, ma jeune amie ; repose-toi sur mes genoux.

Que ton regard est enchanteur!

Que le mouvement de ton sein est vif et délicieux sous la main qui le presse !

Tu souris, Nahandove, ô belle Nahandove !

Tes baisers pénètrent jusqu'à l'âme ;

tes caresses brûlent tous mes sens ; arrête, ou je vais mourir. Meurt-on de volupté,

Nahandove, ô belle Nahandove?

Le plaisir passe comme un éclair. Ta douce haleine s'affaiblit, tes yeux humides se referment, ta tête se penche mollement, et tes transports s'éteignent dans la langueur. Jamais tu ne fus si belle, Nahandove, ô belle Nahandove ! [...]*

Tu pars, et je vais languir dans les regrets et les désirs.

Je languirai jusqu'au soir.

Tu reviendras ce soir, Nahandove, ô belle Nahandove !

* Note : Ravel a supprimé la phrase suivante : "Que le sommeil est délicieux dans les bras d'une maîtresse ! Moins délicieux pourtant que le réveil. » 


\section{Pierre Louÿs (1894) et Claude Debussy (1899) : «La flûte de Pan»}

Pour le jour des Hyacinthies, il m'a donné une syrinx faite de roseaux bien taillés, unis avec la blanche cire qui est douce à mes lèvres comme le miel.

Il m'apprend à jouer, assise sur ses genoux; mais je suis un peu tremblante. Il en joue après moi, si doucement que je l'entends à peine.

Nous n'avons rien à nous dire, tant nous sommes près l'un de l'autre ;

mais nos chansons veulent se répondre, et tour à tour nos bouches s'unissent sur la flûte.

Il est tard ; voici le chant des grenouilles vertes qui commence avec la nuit. Ma mère ne croira jamais que je suis restée si longtemps à chercher ma ceinture perdue.

\section{Paul Verlaine (1869) et Claude Debussy (1888) :}

"Le Faune "

Un vieux faune de terre cuite

Rit au centre des boulingrins,

Présageant sans doute une suite

Mauvaise à ces instants sereins

Qui m'ont conduit et t'ont conduite,

- Mélancoliques pèlerins, -

Jusqu'à cette heure dont la fuite

Tournoie au son des tambourins. 